\title{
Prevalence of occult inflammatory bowel disease in ankylosing spondylitis
}

\author{
PATRICK B. COSTEllo, JORGE A. AlEA, Alastair C. KENNEDy, \\ ROBERT T. MCCLUSKEY, AND FLOYD A. GREEN
}

From the Departments of Medicine and Pathology, State University of New York at Buffalo, and the Veterans Administration Hospital, Buffalo, New York, USA

SUMMARY Fifty-five patients with ankylosing spondylitis and 16 control patients matched for sex and age were examined for evidence of occult inflammatory bowel disease. In all patients evaluation included history and physical examination, barium enema, sigmoidoscopy, and rectal biopsy. The results of this study suggest that there is no increased prevalence of occult inflammatory bowel disease in patients with ankylosing spondylitis.

The association between chronic inflammatory bowel disease and ankylosing spondylitis is well established. ${ }^{1}$ Although the prevalence in reported series varies widely, studies of index patients with Crohn's disease and ulcerative colitis suggest an approximate frequency of spondylitis of $20 \%$ and $11 \%$, respectively. ${ }^{2-4}$ In the case of Crohn's disease the association is strongest when the large bowel is involved. ${ }^{45}$

When the relationship between chronic inflammatory bowel disease and ankylosing spondylitis is looked at conversely, an equally wide range of prevalence data has been reported. Estimates of the frequency of chronic inflammatory bowel disease in patients with ankylosing spondylitis vary between $2.4 \%$ and $18 \%,{ }^{6-10}$ with ulcerative colitis occurring more frequently than Crohn's disease.

Regardless of the analytical approach, the validity of the results is subject to various methodological problems. Several selection factors are important, including the status of the patient population with regard to the presence or absence of symptoms; the likelihood of bias resulting from overascertainment, i.e., the increased chance of recognising a second disease in the follow-up of a chronic disease diagnosed previously; and other factors leading to poor rates of completing the follow-up.

In the case of prevalence studies of chronic inflammatory bowel disease in ankylosing spondylitis wide variation in the thoroughness of the

Accepted for publication 31 August 1979

Correspondence to Patrick B. Costello, MD, Department of Medicine, State University of New York at Buffalo, 3495 Bailey Avenue, Buffalo, New York 14215, USA. gastrointestinal examinations employed and poorly defined diagnostic criteria in some studies further complicate interpretation of the results.

Perhaps a more germane question asked about a group of patients with ankylosing spondylitis is whether they have an increased prevalence of occult bowel disease. Previous studies which provide such data suggest the existence of a small but significant subpopulation of ankylosing spondylitic patients with asymptomatic chronic inflammatory bowel disease, ${ }^{7-9}$ prompting one group of authors to recommend that all ankylosing spondylitic patients be screened for possible occult bowel disease. ${ }^{9} \mathrm{~A}$ recent report, with a more complete gastrointestinal evaluation of symptomatic and asymptomatic ankylosing spondylitic patients, does not confirm this finding. ${ }^{10}$

The present controlled study was undertaken to evaluate the frequency of occult chronic large bowel disease in an unselected series of spondylitic patients attending the Buffalo Veterans Administration Hospital outpatient clinic.

\section{Materials and methods}

Sixty-eight patients with ankylosing spondylitis who had been seen at the Buffalo Veterans Administration Hospital in the 10 years prior to the inception of the study were invited to participate in the investigation. Fifty-five patients entered the study and were fully evaluated, a completion rate of over $80 \%$. Nine of these patients were known to have well established diagnoses of chronic inflammatory bowel disease, including 3 patients with Crohn's 
disease and 6 with ulcerative colitis. Most of these patients had been referred to us, usually from a gastroenterology clinic. A control group of 16 consecutive patients admitted to hospital with convulsive disorders was chosen because of similarities to the experimental group in such characteristics as age range, sex, and approximate frequency of alcoholism. In the majority of control cases the seizure disorder was secondary to withdrawal of alcohol, and of barbiturates in a few. In the remaining cases focal structural abnormalities were present on electroencephalographic recordings.

The experimental and control groups underwent identical evaluations. Each patient had a complete history and physical examination by one observer (F.G.) with particular attention paid to symptoms and/or signs referrable to the musculoskeletal systems. Neck flexion and chest expansion were tested, as described by Potter ${ }^{11}$ and Wright and Moll. ${ }^{12}$ Additional rheumatological evaluation consisted of radiological examination of the axial skeleton in all cases and of peripheral joints if indicated by positive symptoms or signs. Radiological criteria of sacroiliitis conformed with categories no. 3 or 4 as described by Bennett and Burch. ${ }^{13}$

Gastrointestinal evaluation in all patients included noting the presence or absence of abdominal pain and diarrhoea with or without bloody stools. Barium enemas were performed under standard conditions. All patients underwent successful proctosigmoidoscopic examination by one operator (J.A.). During the procedure a biopsy was obtained from the rectum by a consistent punch technique at the level of Houston's valves, $10 \mathrm{~cm}$ proximal to the anus. Patients were prepared for endoscopy with 1 bisacodyl (Dulcolax) suppository an hour before the examination; enemas were not used. No serious complications resulted from the procedure.

The following diagnostic criteria were adhered to in this investigation. All spondylitic patients had a history and/or physical examination compatible with the diagnosis and unequivocal radiological evidence of sacroiliitis. Old films were reviewed when necessary, and oblique. Ferguson, and occasionally tomographic views were used to clarify potentially misleading disorientated views and to confirm the diagnosis in a few problematic cases, such as an elderly patient with findings also consistent with degenerative joint disease or osteitis condensans ilii. The spondylitic patients thus conformed to the New York diagnostic criteria for definite ankylosing spondylitis. ${ }^{13}$ Any information gained from proctosigmoidoscopic and barium enema examinations was evaluated for diagnostic criteria as previously published. ${ }^{14-16}$

Biopsy specimens were coded and interpreted by a pathologist (R.M.) without knowledge of other findings in the case. Each specimen was evaluated semiquantitatively for the presence and numbers of neutrophils, eosinophils, plasma cells, macrophages, and lymphocytes; the numbers were arbitrarily graded on a scale of 0 to $3+$; grades of 2 or $3+$ were considered to represent excessive accumulations. Each specimen was also examined for the presence of granulomas or microabscesses. After the initial histopathological diagnosis, a coded subsample of all suspicious changes in the control group was reinterpreted blindly by the same pathologist and the original interpretation was consistently confirmed.

Laboratory evaluation performed in both groups included serum or blood measurement of completed blood count and smear, erythrocyte sedimentation rate (ESR), reticulocyte count, platelet count, serum iron/total iron binding capacity, serum carotene, serum protein electrophoresis, serum immunoelectrophoresis, rheumatoid factor, and antinuclear antibody titres. Statistical analysis was by $t$ tests of the differences in prevalence rates between the experimental and control groups, both collectively and for individual measurements.

\section{Results}

A full evaluation of 55 patients with ankylosing spondylitis and 16 control patients was completed. Of the 55 patients in the experimental group 9 patients, as outlined above, had previously established chronic inflammatory bowel disease. Three of them had Crohn's disease and 6 had ulcerative colitis. For the purposes of this study these 9 patients with bowel disease and related spondylitis were not included in the analysis detailed below.

Table 1 describes the clinical features of the 2 groups of patients, consisting of 46 patients with apparently uncomplicated ankylosing spondylitis and 16 control patients. As can be seen, they were well matched for age and sex. The average duration of spondylitis was 21 years. Decreased neck flexion and chest expansion was detected in $72 \%$ and $76 \%$, respectively, of the spondylitic group. There was clinically apparent involvement of one or both hip joints in $28 \%$ of the patients. Peripheral joint involvement was present in $46 \%$ of the patients with ankylosing spondylitis. All patients in the spondylitic group had clear evidence of sacroiliac joint involvement, and radiographical evidence of hip and peripheral joint involvement was found in $15 \%$ and $23 \%$, respectively, of the spondylitic group.

None of the control patients had rheumatological abnormalities. There were no significant differences between the spondylitic and control groups with regard to levels of haemoglobin concentration, 
haematocrit, reticulocyte count, total white blood cell count, platelet count, mean sedimentation rate, serum iron and total iron binding capacity, serum carotene, immunoglobulins, antinuclear antibody, and rheumatoid factor titres. Similarly, there were no important differences in laboratory data between the 9 patients with known chronic inflammatory bowel disease and either the spondylitic or control population. The raised mean ESR of the control group is probably due to the hepatic dysfunction which was present in many of these patients early in their hospital course.

Clinical examination of the gastrointestinal system revealed nonspecific findings. No patients had abnormalities on physical examination of the abdomen. Of the 46 spondylitic patients, 9 had intestinal symptoms. Four of these patients complained of dyspepsia or nonspecific epigastric pain, and all 4 had normal barium enema and sigmoidoscopic examinations. The remaining 5 patients complained of mild to moderate intermittent diarrhoea with or without constipation; of these 5 patients 4 had abnormal barium enemas, one with diverticulosis and 3 with nondiagnostic patterns such as 'spasm' or 'smooth pattern'. None of the patients in the control group had symptoms or showed abnormalities on barium enema or sigmoidoscopy.

Isolated abnormalities of the barium enema or sigmoidoscopy occurred in 7 of the remaining spondylitic group. Three of these patients had hyperaemia of the distal colon. Contrast studies of the large bowel revealed diverticulosis in 3 patients and spasticity in the seventh. Finally, in 1 patient a sigmoid colon polyp was found on endoscopy, and diverticuli were noted on the barium enema examination.

Table 2 details the results of interpretation of the biopsy specimens. These results were derived from the semiquantitative grading of individual biopsy specimens. Accumulations of inflammatory cells that were considered to be excessive are listed. As can be seen, increased numbers of leucocytes, especially neutrophils, eosinophils, and macrophages, were seen slightly more frequently in the spondylitic group than the control group, but the differences were not statistically significant. No patients from either group had abnormal accumulations of plasma cells. One spondylitic patient had microabscesses in the biopsy specimen; these were not found in any control patients.

$t$ tests were not significant when differences in prevalence of all the abnormalities between the spondylitics and controls were compared. Although randomnly mixed within the whole group of specimens, biopsies from all 9 patients with chronic inflammatory bowel disease were recognised by the pathologist as abnormal.

\section{Discussion}

This study was designed to investigate the prevalence of occult large bowel disease in a group of spondy-

Table 1 Clinical features of spondylitic patients and control subjects

\begin{tabular}{|c|c|c|}
\hline & Spondylitis & Control \\
\hline $\begin{array}{l}\text { Number } \\
\text { Sex: male } \\
\quad \text { female } \\
\text { Age, Yr (mean } \pm \text { SD) } \\
\text { Duration spondylitis, yr (mean) } \\
\text { Decreased lateral neck flexion } \\
\text { Decreased chest expansion } \\
\text { Signs and symptoms, hip joint } \\
\text { Signs and symptoms, any other peripheral joint } \\
\text { Radiography: abnormal sacroiliac joints } \\
\quad \text { abnormal hip joint } \\
\text { abnormal other peripheral joint } \\
\text { Laboratory: haemoglobin (g/dl, mean } \pm S D) \\
\text { haematocrit (g/dl, mean } \pm S D) \\
\text { white blood cell count }(\times 109 / 1, \text { mean } \pm S D) \\
\text { erythrocyte sedimentation rate, } \\
\text { Westergren (mm/hr, mean } \pm S D)\end{array}$ & $\begin{array}{l}46 \\
45 \\
1 \\
47 \cdot 6 \pm 12 \cdot 4 \\
21 \\
33(71 \cdot 7 \%) \\
35(76 \cdot 1 \%) \\
13(28 \cdot 2 \%) \\
21(45 \cdot 6 \%) \\
46(100 \%) \\
7(14 \cdot 6 \%) \\
11(22 \cdot 9 \%) \\
14 \cdot 2 \pm 2 \cdot 0 \\
44 \cdot 8 \pm 4 \cdot 3 \\
6 \cdot 82 \pm 1 \cdot 79 \\
34 \cdot 1 \pm 25 \cdot 7\end{array}$ & $\begin{array}{l}16 \\
15 \\
1 \\
49 \cdot 0 \pm 10 \cdot 1 \\
- \\
- \\
- \\
- \\
- \\
- \\
13 \cdot 9 \pm 1 \cdot 4 \\
44 \cdot 2 \pm 4 \cdot 0 \\
7 \cdot 42 \pm 2 \cdot 54 \\
36 \cdot 0 \pm 31.9\end{array}$ \\
\hline
\end{tabular}

Table 2 Spondylitic and control subjects with abnormal cellular infiltrates on rectal biopsy.

\begin{tabular}{|c|c|c|c|c|c|c|c|}
\hline \multirow[t]{2}{*}{ Group } & \multicolumn{7}{|c|}{ Number with abnormal accumulation } \\
\hline & $N$ & Neutrophils & Eosinophils & Macrophages & Lymphoid cells & Plasma cells & Microabscesses \\
\hline $\begin{array}{l}\text { Spondylitis } \\
\text { Control }\end{array}$ & $\begin{array}{l}46 \\
16\end{array}$ & $\begin{array}{l}4(8 \cdot 7)^{*} \\
2(12 \cdot 5)\end{array}$ & $\begin{array}{l}6(13 \cdot 0) \\
2(12 \cdot 5)\end{array}$ & $\begin{array}{l}4(8 \cdot 7) \\
1(6 \cdot 2)\end{array}$ & $\begin{array}{l}1(2 \cdot 2) \\
0(0)\end{array}$ & $\begin{array}{l}0(0) \\
0(0)\end{array}$ & $\begin{array}{l}1(2 \cdot 2) \\
0(0)\end{array}$ \\
\hline
\end{tabular}

* Numbers in parentheses are percentages. 
litic patients. In planning the study design an attempt was made to optimise control of selection factors and minimise other sources of built-in bias. All patients with ankylosing spondylitis seen in our general rheumatology clinic were asked to participate in the study. The completion rate of $80 \%$ achieved in this study is substantially better than in previous prevalence studies of the association between ankylosing spondylitis and bowel disease. ${ }^{89}$ The high completion rate in our study was probably due in large part to the fact that a majority of the spondylitic patients had service-connected pensions.

The $16 \cdot 3 \%$ prevalence rate of chronic inflammatory bowel disease in our initial spondylitic population of 55 patients is in agreement with reports by Jayson et al. ${ }^{9}$ and Jayson and Bouchier, ${ }^{8}$ with rates of $17 \%$ and $18 \%$ respectively. All but 1 of their patients with chronic inflammatory bowel disease had ulcerative colitis. It is possible that results of the above studies as well as our own may be biased by the increased chance of discovering a second disease in a population with an already established chronic disease. In the 2 cited studies by Jayson et al., ${ }^{9}$ the authors also suggested that bias may have been introduced by selection factors favouring the inclusion of symptomatic patients.

Our study demonstrates no increase in prevalence of occult large-bowel disease in a group of spondylitic patients compared to an appropriately matched control group who were studied by clinical, radiological, and biopsy procedures. Three patients from the control group had 'abnormal' cellular infiltrates on rectal biopsy. It is unlikely that such findings could be related to alcohol abuse, since it has been shown that, even as far distally as the small bowel, biopsies were normal by light microscopy in chronic alcoholics both before and after ethanol ingestion. ${ }^{17}$ No cases of asymptomatic bowel disease in this series of patients were uncovered by our investigations, in contrast to Jayson et al. ${ }^{9}$ who reported that of 8 patients diagnosed as having chronic bowel disease 3 were symptom-free. However, the diagnosis of bowel disease in none of these patients fulfilled all the authors' diagnostic criteria. In their studies, as well as our own, upper gastrointestinal examinations were not performed. Thus it is possible that some cases of enteritis were overlooked.

The failure to elucidate unsuspected bowel disease in this paper is in agreement with the recent study by Meuwissen et al. ${ }^{10}$ This well-designed study of 79 spondylitic patients using the same procedures as our study, including rectal biopsies in selected subgroups as well as upper gastrointestinal examination, failed to reveal any hidden cases of chronic inflammatory bowel disease. Three patients $(3.8 \%)$ had previously diagnosed Crohn's disease. It is not known whether additional cases of large bowel disease might have been uncovered if more of their patients had had barium enema examination and biopsies carried out.

In conclusion, this study was designed to detect any possible occult inflammatory large bowel disease in a group of patients with ankylosing spondylitis. No such tendency was demonstrated. Meuwissen et al. ${ }^{10}$ while not directly addressing this question, provide data which support our observation. Thus it seems unlikely that any significant prevalence of occult bowel disease exists in patients with ankylosing spondylitis, and intensive investigation of their gastrointestinal tract is unlikely to prove fruitful.

\section{References}

1 Haslock I, Wright V. The arthritis associated with intestinal disease. Bull Rheum Dis 1973-4; 24: 750-5.

2 Wright V, Watkinson G. The arthritis of ulcerative colitis. Br Med J 1965; 2: 670-5.

${ }^{3}$ Hammer B, Ashurst P, Naish J. Diseases associated with ulcerative colitis and Crohn's disease. Gut 1968; 9: 17-21.

4 Haslock I, Wright V. The musculoskeletal complication of Crohn's disease. Medicine 1973; 52: 217-25.

5 Cornes J S, Stecher M. Primary Crohn's disease of the colon and rectum. Gut 1961; 2: 189-201.

6 Wilkinson M, Bywaters E G L. Clinical features and course of ankylosing spondylitis. Ann Rheum Dis 1958 17: 209-28.

7 McBride J A, King M J, Baikie A G, Crean G P, Sircus W. Ankylosing spondylitis and chronic inflammatory diseases of the intestines. $\mathrm{Br}$ Med $J$ 1963; 2: 483-6.

8 Jayson M I V, Bouchier I A D. Ulcerative colitis with ankylosing spondylitis. Ann Rheum Dis 1968; 27: 219-24.

9 Jayson M I V, Salmon P R, Harrison W J. Inflammatory bowel disease in ankylosing spondylitis. Gut 1970; 11: 506-11.

10 Meuwissen S G M, Dekker-Saeys B J, Agenant D, Tytgat G N D. Ankylosing spondylitis and inflammatory bowel disease. Ann Rheum Dis 1978; 37: 30-2.

11 Potter T A. In: Hollander J L, McCarty D J Jr, eds. Arthritis and Allied Conditions, 8th ed. London: Henry Kimpton, 1972: 1453.

12 Wright V Moll J M H. Seronegative Polyarthritis. Amsterdam: Elsevier/North-Holland Biomedical Press, $1976 ; 163$

13 Bennett P H, Burch T A. New York symposium on population studies in the rheumatic diseases; new diagnostic criteria Bull Rheum Dis 1967; 17: 453-8.

14 Palmer W L. Chronic ulcerative colitis. Gastroenterology $1948 ; 10$ : 767-81.

15 Fennessy J J, Sparberg M, Kirsner J B. Early roentgen manifestations of mild ulcerative colitis and proctitis. Radiology 1966; 87: 848-58.

16 Geffen N, Darnborough A, de Dombal F T, Watkinson G, Goligher J C. Radiological signs of ulcerative colitis: assessment of their reliability by means of observer variation studies. Gut 1968; 9: 150-6.

17 Rubin E, Rybak B J, Lindenbaum J, Gerson C D, Walker G. Lieber C S. Ultrastructural changes in the small intestine induced by ethanol. Gastroenterology 1972; 63: 801-14. 